\title{
Structural study of conventional and bulk metallic glasses during annealing
}

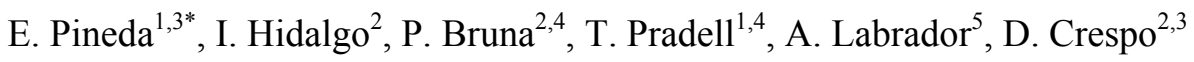

${ }^{1}$ Departament de Física i Enginyeria Nuclear, ESAB \& ${ }^{2}$ Departament de Física Aplicada, EPSC, Universitat Politècnica de Catalunya, Avda. del Canal Olímpic s/n, 08860 Castelldefels, Spain

${ }^{3}$ Centre de Recerca de l'Aeronutica i de l'Espai $\&{ }^{4}$ Centre de Recerca en Nanoengineyeria, UPC ${ }^{5}$ LLS - BM16, ESRF, 38043-Grenoble, France

*Corresponding author:

Eloi Pineda

eloi.pineda@upc.edu

Tel. (34) 935521141

Fax. (34) 935521001

\begin{abstract}
Metallic glasses with conventional glass-forming ability (Al-Fe-Nd, Fe-Zr-B, Fe-B-Nb compositions) and bulk metallic glasses ( $\mathrm{Ca}-\mathrm{Mg}-\mathrm{Cu}$ compositions) were studied by synchrotron X-ray diffraction during annealing throughout glass transition and crystallization temperatures. The analysis of the first diffraction peak position during the annealing process allowed us to follow the free volume change during relaxation and glass transition. The structure factor and the radial distribution function of the glasses were obtained from the X-ray measurements. The structural changes occurred during annealing are analyzed and discussed.
\end{abstract}

Keywords: Amorphous materials / metallic glasses / rapid-solidification, quenching / atomic scale structure / X-ray diffraction. 


\section{Introduction}

The study of the mechanical properties of amorphous or glassy alloys has become an active field of research, as the improvement of these properties promises future applications of metallic glasses (MG) as structural materials. The ductility of these materials is one of the key properties determining their applicability [1]. Recently, molecular dynamics simulations showed that the plastic deformation process is closely related to the presence of certain configurations in the atomic structure [2]; open atomic configurations facilitate plastic flow and the initiation of shear bands, hence improving ductility. Other theoretical studies relate changes of the elastic constants to small variations of the mean position and width $\sigma$ of the first-neighbour peak of the atomic radial distribution function (RDF) [3]. A reduction of $\sigma$, as expected after structural relaxation, implies an increase of the shear to bulk modulus ratio thus stiffening the material and reducing ductility. Experimentally, some MG alloys show a transition from ductile to brittle behaviour after structural relaxation [4], as the densification occurred during relaxation involves the reduction of open atomic configurations thus blocking plastic flow. In the super-cooled liquid state, the temperature-viscosity behaviour can be interpreted in terms of free volume change in the liquid. In the amorphous solid state, the free volume quenched-in during the glass transition characterizes the glassy state, and can be reduced because of structural relaxation during annealing treatments. The presence of free-volume, that from a microscopic point of view implies more open atomic arrangements, has also been shown to facilitate homogeneous plastic deformation [5].

The study of the atomic structure of MG alloys and its evolution during annealing processes is then important for understanding the mechanical properties of these materials and for finding strategies to improve them. In this paper we present in-situ synchrotron X-ray diffraction measurements that allow us to study the thermal dilatation, the free volume change and the crystallization occurred during annealing of MGs. Results for the radial distribution function and volume change during the heating treatments are analyzed and discussed.

\section{Experimental details}


Metallic glass samples were obtained as ribbons by melt-spinning; their glass transition and crystallization were previously analysed by DSC measurements. The compositions studied in the present work are listed in table 1. The Al and Fe-based alloys are conventional metallic glasses with low glass forming ability while the $\mathrm{Ca}-\mathrm{Mg}-\mathrm{Cu}$ alloys are bulk metallic glasses with large glass stability. The thickness of the ribbons ranges from 30 to $140 \mu \mathrm{m}$ depending on the alloy. The X-ray measurements were performed at the BM16 line of the European Synchrotron Radiation Facility (ESRF). The ribbons were cut in $0.3 \times 1 \mathrm{~cm}$ pieces and introduced into a Linkam hot stage, fixed in a metallic washer. The diffracted intensity was collected by a 2dimensional ADSC Q210r CCD detector perpendicular to the incident beam in transmission geometry. The time-resolution of the X-ray detector allowed us to follow the structural changes of the samples throughout the annealing process. Various annealing protocols with heating and cooling rates of $10-20 \mathrm{~K} / \mathrm{min}$ were applied, and spectra were acquired every $5 \mathrm{~s}$. The annealing was performed under nitrogen flow in order to minimize the oxidation of the samples.

The radiation energy used was of $15 \mathrm{keV}$ with a corresponding wave length of $0.826 \AA$. The spectra used to determine the RDF of the alloys at different points during the annealing protocols were treated separately. The total intensity elastically scattered by the samples $I(Q)$ was obtained subtracting background and applying absorption, multiple scattering, and inelastic Compton scattering corrections. The absorption correction in flat transmission geometry was performed following ref. [6]. The Compton scattering contribution was calculated using the fitting parameters provided in ref. [7] for the different elements. The normalization factor was obtained by the Krogh-Moe-Norman method. From $I(Q)$ the total structure factor $S(Q)$ was obtained by

$$
S(Q)=\frac{I(Q)-\left\langle f^{2}\right\rangle}{\langle f\rangle^{2}}+1
$$

and the average atomic factors of the alloys $\langle f\rangle$ were calculated using the tabulated values in refs. $[8,9]$ compiled in the DABAX data base [10]. The reduced radial distribution function was then obtained by Fourier transformation 
$G(r)=4 \pi r \rho_{0}[g(r)-1]=\frac{2}{\pi} \int_{0}^{Q_{\max }} Q[S(Q)-1] \sin (Q r) d Q$

where $g(r)$ is the pair distribution function, and the radial distribution function is then calculated

as $R D F(r)=4 \pi r^{2} \rho_{0} g(r)$. The experimental setup allows the measurement of the diffracted intensity up to $Q_{\max }=8 \AA^{-1}$. This low value of $Q_{\max }$ impedes a proper normalization of $I(Q)$ and it is expected to broaden artificially the peaks of the calculated RDFs $[6,11]$.

\section{Results and discussion}

The intensity diffracted by the samples was recorded at temperature intervals of $0.833 \mathrm{~K}$ and $1.67 \mathrm{~K}$ for heating or cooling rates of respectively $10 \mathrm{~K} / \mathrm{min}$ and $20 \mathrm{~K} / \mathrm{min}$; this allows us to follow accurately the structural changes all along the annealing protocols. The onset of crystallization can be well determined due to the high resolution of the synchrotron measurements. Figure 1 shows the appearance of peaks for the $\mathrm{Al}_{90} \mathrm{Fe}_{5} \mathrm{Nd}_{5}$ alloy due to the primary crystallization of crystalline Al. These crystalline peaks are noticed since $T=188.1{ }^{\circ} \mathrm{C}$, corresponding to the diffraction spectrum depicted with dashed line in figure 1. Previous DSC measurements of the same alloy (figure 1 inset) gave a primary crystallization temperature of $T_{\mathrm{x} 1}=185^{\circ} \mathrm{C}$ for the same heating rate.

Although crystallization is observed from the initial stages, the structural changes in the glassy state are hardly perceived in the diffracted intensities or the corresponding structure factors. Figure 2 shows the main diffraction peak obtained for the $\mathrm{Al}_{90} \mathrm{Fe}_{5} \mathrm{Nd}_{5}$ at different stages during an annealing cycle, $25-175-50-500{ }^{\circ} \mathrm{C}$, with heating and cooling rates of $20 \mathrm{~K} / \mathrm{min}$. As will be shown below there are differences in free volume between the different points of the cycle; however, differences in the structure factor or the RDFs are hardly observed. Figure 3 displays the total structure factor and the corresponding RDF obtained for the as-quenched $\mathrm{Al}_{90} \mathrm{Fe}_{5} \mathrm{Nd}_{5}$ alloy at room temperature; the coordination number from the RDF is of 11.5 . Neither the RDF nor the coordination number show significant changes over the annealing cycle. Previous studies on metallic glasses reported typically small changes of about $2 \%$ or less in the area of the first nearest-neighbour peak of the RDF between different glassy states [12, 13]. Concerning to the diffracted intensity, differences between different glassy states are just 
slight variations of the position and width of the main diffraction peak. As already stated, although these are small differences, they may result in important changes in the behaviour of the macroscopic properties.

In this paper we will analyze the variation of the main diffraction peak during the annealing protocols. The variation of the position $Q_{\max }$ of the first diffracted intensity maximum is inversely proportional to the mean inter-atomic spacing. In ref. [14], Yavari et al. showed that the free volume content is homogeneously distributed in the atomic structure of metallic glasses. This implies that the change in the mean atomic volume can be calculated by

$$
\left[\frac{Q_{\max }\left(T_{0}\right)}{Q_{\max }(T)}\right]^{3}=\frac{V(T)}{V\left(T_{0}\right)}
$$

where $V$ is the atomic volume and $T_{0}$ is a temperature reference; in this work $T_{0}$ is the room temperature. The atomic volume change computed using equation (3) is equivalent to the macroscopic volume change obtained by dilatometry $[14,15]$. Figure 4 shows the variation of $Q_{\max }$ for a $\mathrm{Fe}_{80} \mathrm{~B}_{12} \mathrm{Nb}_{7} \mathrm{Cu}_{1}$ alloy; this slight variation can be well determined by fitting the very top of the peak to a parabolic function.

The change in the mean atomic volume of metallic glasses during annealing is expected to result from reversible thermal dilatation and structural changes of the amorphous phase; in the latter case the volume change is irreversible, resulting in a reduction of the free volume content of the glass. Figure 5 shows the $V(T) / V\left(T_{0}\right)$ obtained for two of the studied alloys. For the Fe based alloys $\left(\mathrm{Fe}_{80} \mathrm{~B}_{12} \mathrm{Nb}_{7} \mathrm{Cu}_{1}\right.$ and $\left.\mathrm{Fe}_{89} \mathrm{Zr}_{7} \mathrm{~B}_{4}\right)$ an initial linear thermal dilatation zone is followed by structural relaxation prior to crystallization; this is shown in figure 5 (top) for the $\mathrm{Fe}_{80} \mathrm{~B}_{12} \mathrm{Nb}_{7} \mathrm{Cu}_{1}$ ribbon. The beginning of crystallization is clearly observed as a drastic drop of the main diffraction peak position because of the appearance of a crystalline diffraction peak at a different angle, while the structural relaxation results in the typical departure form the low temperature lineal behaviour usually observed in dilatometry experiments. The observed crystallization temperatures $T_{x}$ coincide with the values obtained by calorimetry for the same alloys; however, the structural relaxation was not detected in the previous analysis of the DSC measurements. 
Figure 5 (bottom) shows the volume change during a heating-cooling cycle for the $\mathrm{Al}_{90} \mathrm{Fe}_{5} \mathrm{Nd}_{5}$ ribbon. The structural relaxation is not clearly observed in the $\mathrm{Al}$ and Ca-based alloys, with crystallization temperatures much lower than the Fe based alloys (see table 1). In constant heating experiments the $V(T) / V\left(T_{0}\right)$ ratio does not depart from a lineal behaviour until the onset of crystallization. However, after a heating-cooling cycle below the crystallization temperature a reduction of $V(T)$ is clearly observed. For the $\mathrm{Al}_{90} \mathrm{Fe}_{5} \mathrm{Nd}_{5}$ alloy the difference between the as-quenched and the annealed ribbon at $T=50^{\circ} \mathrm{C}$ is of $\Delta V=0.54 \%$, which is similar to the volume changes occurred during structural relaxation in many metallic glasses, and the diffraction intensities obtained at different points of the cycle (see figure 2) do not show evidence of crystallization. The shape of the $V(T) / V\left(T_{0}\right)$ ratio in figure 5 and the results of other studies in Al-based amorphous alloys may suggest some slight degree of crystallization or nuclei formation during the first heating-cooling cycle. This crystallization may be small enough to be below the synchrotron diffraction sensitivity. Either resulting from structural relaxation or initiation of crystallization (many times the two phenomena are closely linked) the volume change of the material is detectable by the analysis of the $Q_{\max }$ position.

Similar experiments were realized for all the alloys in table 1. The crystallization temperatures and coefficients of lineal thermal expansion $\alpha_{\text {th }}$ obtained from the analysis of the $Q_{\max }$ position are listed in table 1 . The values of $\alpha_{\text {th }}$ are similar to the values found in the literature for similar compositions. As already stated, structural relaxation was clearly observed in the Fe-based alloys, which have high crystallization temperatures. For the Al and Ca-based alloys a departure from lineal thermal dilatation behaviour was not observed in the constant heating rate treatments, although irreversible differences in volume were obtained in the heating-cooling-heating cycles. In the Al-based alloy, the irreversible change may be produced by a slight crystallization or crystal nucleation undetectable by X-ray diffraction. For the Cabased alloys, the noticeable structural relaxation found by DSC measurements in previous works [16] suggests the irreversible change to be caused by structural relaxation in to a more stable glassy state. In all the alloys, differences between different glassy states in the $S(Q)$ and the RDF are hardly detectable. 


\section{Conclusions}

The structure of metallic glass ribbons during annealing has been studied by synchrotron X-ray diffraction. For the investigated alloys, structural changes between glassy states are hardly detectable from the total structure factors. The analysis of the main peak position allows to detect structural relaxation, differences in free volume content and the onset of crystallization. Further analysis of the total and partial radial distribution functions at different points of the annealing protocols may unveil the changes in the atomic structure occurred during the process and responsible for the differences in mean atomic volume.

\section{Acknowledgements}

The authors thank Dr. J. Molera from the Universitat de Girona for her help in DSC and dilatometer measurements. Work funded by CICYT, grant MAT2004-01214 and Generalitat de Catalunya, grants 2005SGR00535 and 2005SGR201.

\section{References}

[1] M.F. Ashby, A.L. Greer, Scripta Mater. 54 (2006) 321-326.

[2] M. Wakeda, Y. Shibutani, S. Ogata, J. Park, Intermetallics 15 (2007) 139-144.

[3] E. Pineda, Phys. Rev. B 73 (2006) 104109.

[4] J.J. Lewandowski, W.H. Wang, A.L. Greer, Philos. Mag. Lett. 85 (2005) 77-87.

[5] F. Spaepen, Scripta Mater. 54 (2006) 363-367.

[6] Y. Waseda, The Structure of Non-Crystalline Materials, McGraw-Hill, 1980.

[7] H.H.M. Balyuzi, Acta Cryst. A31 (1975) 600.

[8] D. Waasmaier, A. Kirfel, Acta Cryst. A51 (1995), 416-413.

[9] L. Kissel, B. Zhou, S. C. Roy, S. K.S. Gupta, R. H. Pratt, Acta Cryst. A51 (1995) 271288.

[10] DABAX A Dynamic Database for X-ray Applications. B. Roux and M.S. del Rio, European Synchrotron Radiation Facility.

[11] Th. Proffen, S.J.L. Billinge, T. Egami, D. Louca, Z. Kristallogr. 218 (2003) 132-143.

[12] Y. Waseda, T. Egami, J. Mater. Sci. 14 (1979) 1249. 
[13] H.S. Chen, K.T. Aust, Y. Waseda, J. Mater. Sic. Lett. 2 (1983) 153.

[14] A.R. Yavari, A. Le Moulec, A. Inoue, N. Nishiyama, N. Lupu, E. Matsubara, W.J. Botta, G. Vaughan, M. Di Michiel, A. Kvick, Acta mater. 53 (2005) 1611-1619.

[15] D.V. Louzguine, A.R. Yavari, K. Ota, G. Vaughan, A. Inoue, J. Non-Cryst. Solids 351 (2005) 1639-1645.

[16] E. Pineda, Y. Zhang, A.L. Greer, J. Alloys and Compounds 434-435 (2007) 145-148. 


\begin{tabular}{|l|c|c|}
\hline Alloy Composition (at.\%) & $T_{x 1}\left({ }^{\circ} \mathrm{C}\right)$ & $\alpha_{\text {th }}\left(\mathrm{x} 10^{-5} \mathrm{~K}^{-1}\right)$ \\
\hline $\mathrm{Al}_{90} \mathrm{Fe}_{5} \mathrm{Nd}_{5}$ & 175 & 2.19 \\
$\mathrm{Ca}_{28.7} \mathrm{Mg}_{16.9} \mathrm{Cu}_{54.4}$ & 140 & 2.15 \\
$\mathrm{Ca}_{28.5} \mathrm{Mg}_{26.5} \mathrm{Cu}_{45.0}$ & 145 & 1.66 \\
$\mathrm{Fe}_{89} \mathrm{Zr}_{7} \mathrm{~B}_{4}$ & 515 & 1.01 \\
$\mathrm{Fe}_{80} \mathrm{~B}_{12} \mathrm{Nb}_{7} \mathrm{Cu}_{1}$ & 490 & 1.29 \\
\hline
\end{tabular}

Table 1.

Composition of the studied alloys and their crystallization temperature and coefficient of lineal expansion calculated from the variation of $Q_{\max }$. Crystallization temperatures are determined at $20 \mathrm{~K} / \mathrm{min}$. 


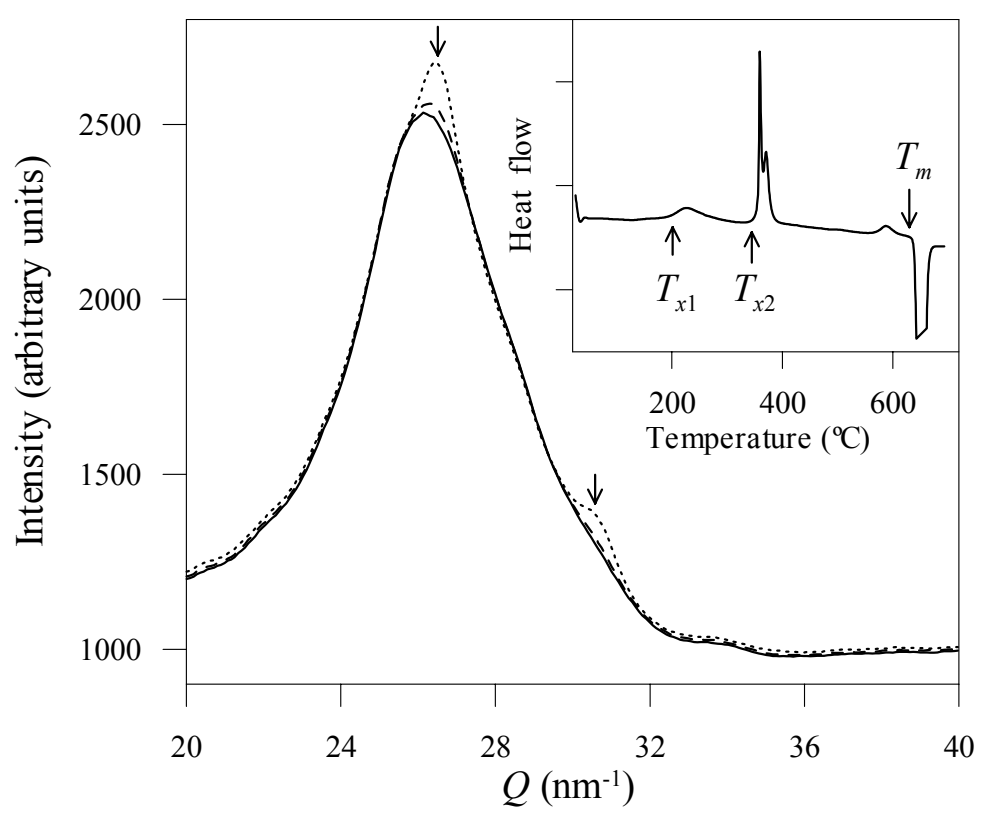

Figure 1.

Main diffraction peak of melt spun $\mathrm{Al}_{90} \mathrm{Fe}_{5} \mathrm{Nd}_{5}$ alloy at 3 different temperatures during annealing with a heating rate of $20 \mathrm{~K} / \mathrm{min}$. The emergence of two peaks of crystalline $\mathrm{Al}$ is observed. Solid line: $T=164.5^{\circ} \mathrm{C}$. Dashed line: $T=188.1^{\circ} \mathrm{C}$. Dotted line: $T=212.3{ }^{\circ} \mathrm{C}$. Inset: DSC curve for $\mathrm{Al}_{90} \mathrm{Fe}_{5} \mathrm{Nd}_{5}$ alloy. 


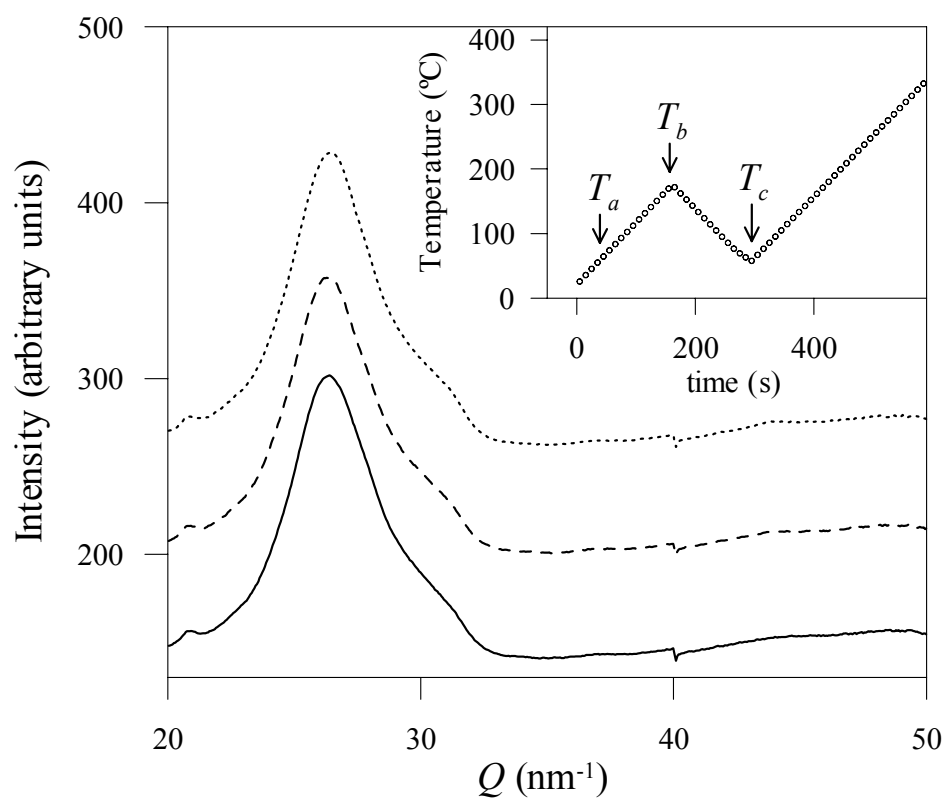

Figure 2 .

Main diffraction peak of melt spun $\mathrm{Al}_{90} \mathrm{Fe}_{5} \mathrm{Nd}_{5}$ alloy at 3 points of the annealing protocol detailed in the inset. Solid line: $T_{a}=59.8^{\circ} \mathrm{C}$. Dashed line: $T_{b}=174.6{ }^{\circ} \mathrm{C}$. Dotted line: $T c=57.8^{\circ} \mathrm{C}$. 


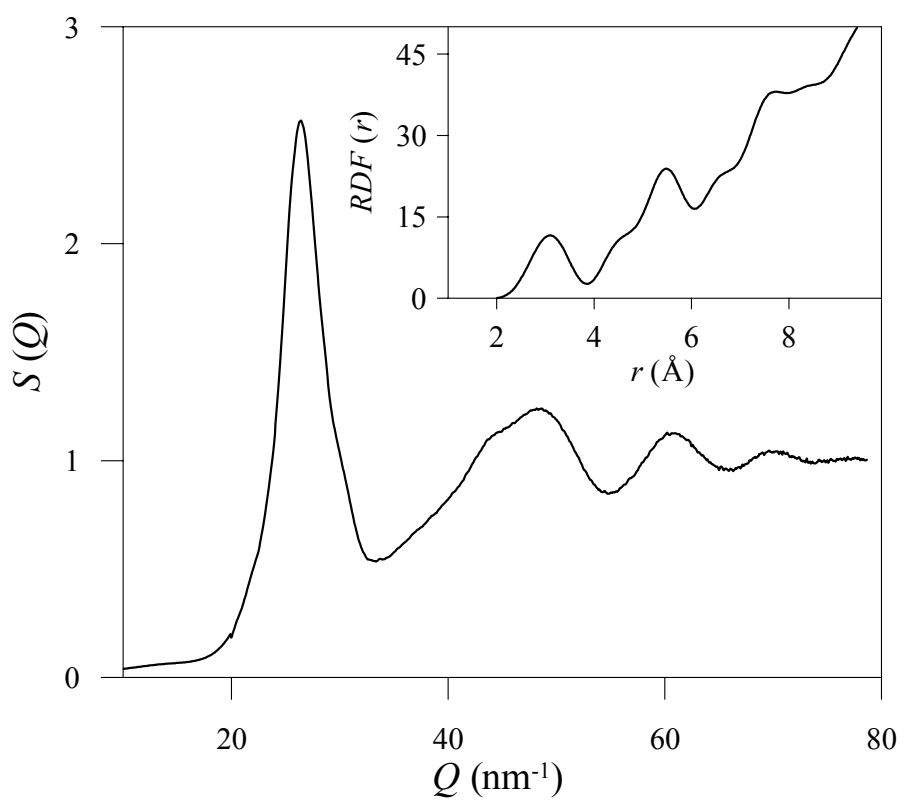

Figure 3.

Total structure factor $S(Q)$ and the corresponding radial distribution function $R D F(r)$ for the $\mathrm{Al}_{90} \mathrm{Fe}_{5} \mathrm{Nd}_{5}$ alloy at room temperature. 


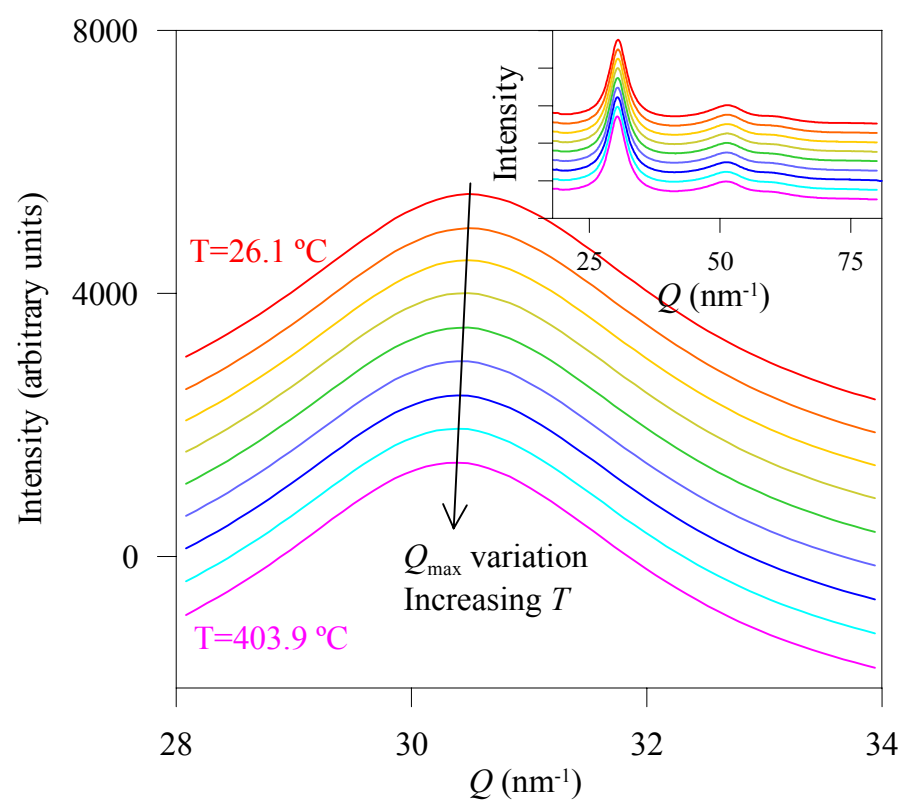

Figure 4.

Variation of the position of the main diffraction peak during annealing for a melt-spun $\mathrm{Fe}_{80} \mathrm{~B}_{12} \mathrm{Nb}_{7} \mathrm{Cu}_{1}$ alloy. Inset: Complete diffracted spectra at the same temperatures. 


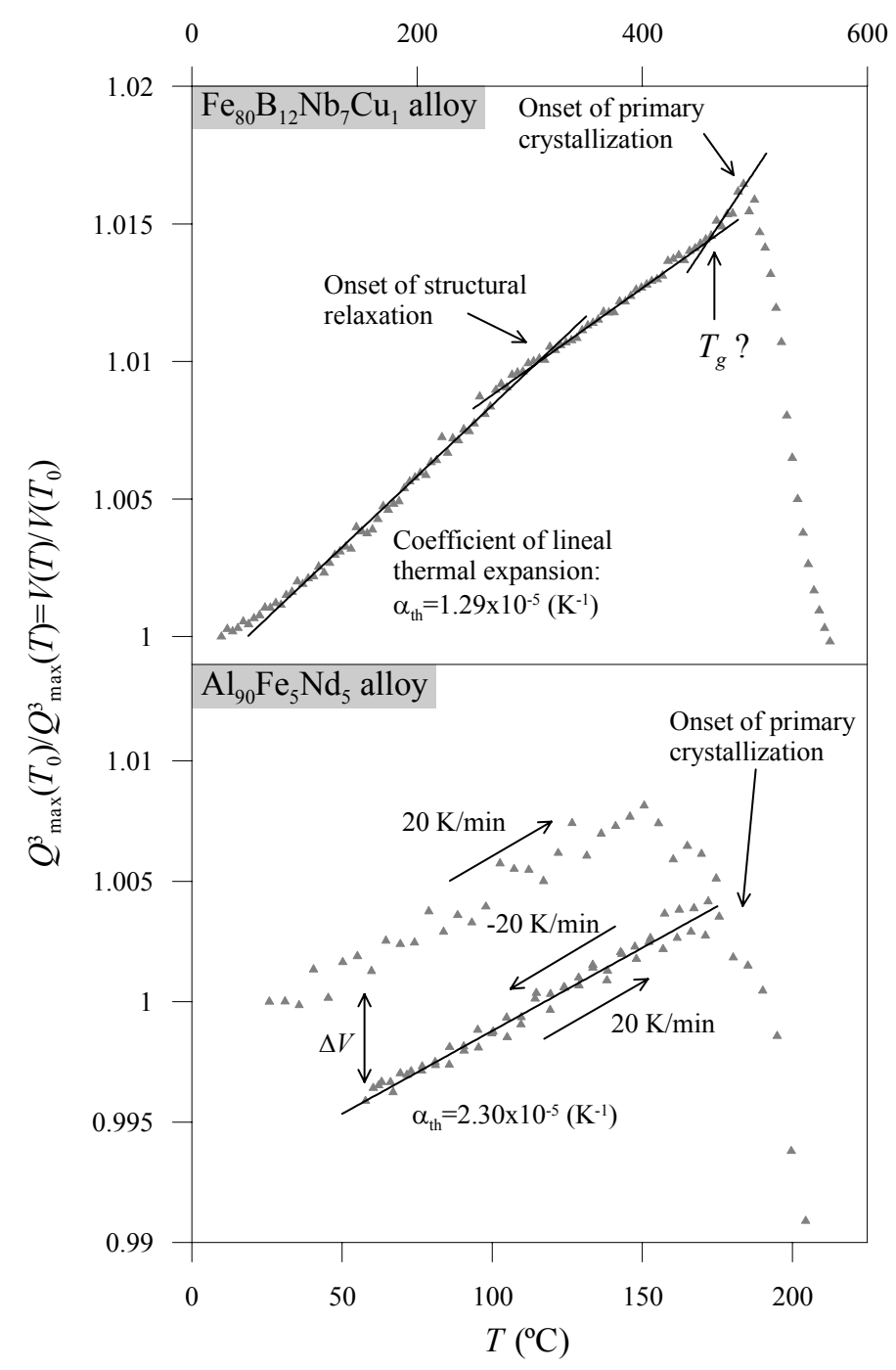

Figure 5.

Mean atomic volume change of amorphous metallic ribbons during annealing protocols. Top: $\mathrm{Fe}_{80} \mathrm{~B}_{12} \mathrm{Nb}_{7} \mathrm{Cu}_{1}$ alloy during a heating protocol at $20 \mathrm{~K} / \mathrm{min}$. Bottom: $\mathrm{Al}_{90} \mathrm{Fe}_{5} \mathrm{Nd}_{5}$ alloy during a heating-cooling-heating cycle at $20 \mathrm{~K} / \mathrm{min}$. 